\title{
INTRANASAL TOPIRAMATE POLYMERIC NANOPARTICLES FOR EPILEPSY: IN VITRO AND EX- VIVO INVESTIGATION
}

\author{
PRAVIN KUKUDKAR, SONIYA RAHATE, RASHMI TRIVEDI, MILIND UMEKAR, JAYSHREE TAKSANDE* \\ Department of Pharmaceutics, Shrimati Kishoritai Bhoyar College of Pharmacy, New Kamptee, Nagpur (M. S.), India 441002 \\ Email: jayabtaksande@gmail.com
}

Received: 09 Mar 2020, Revised and Accepted: 30 Jun 2020

\begin{abstract}
Objective: The presence of tight junctions in blood-brain barrier creates a major problem for the delivery of drugs and severely affects adequate therapeutic concentration to reach the brain. For the treatment of epilepsy, oral route of administration is most convenient but shows delayed absorption. Moreover, in emergency cases, parenteral administration is not possible as it requires medical assistance. Thus, an alternative route of drug delivery is highly desirable for an effective outcome.

Methods: In the present study, bioadhesive chitosan nanoparticles of topiramate for intranasal administration were prepared by ionotropic gelation method using chitosan as bioadhesive polymer and sodium tripolyphosphate as the crosslinking agent. The prepared nanoparticles were evaluated for physicochemical properties like particle size, surface morphology, drug content, entrapment efficiency, thermal behavior and crystallinity, in vitro drug diffusion, ex vivo bio adhesion, and ex vivo biocompatibility studies in excised sheep nasal mucosa.

Results: Differential scanning calorimetry and x-ray diffraction study showed molecular dispersion of drug into the polymer matrices and conversion of it into an amorphous form. Nanoparticles obtained were discrete in nature (size $313.5 \mathrm{~nm}$ ) and appropriate for intranasal administration. The topiramate nanoparticles revealed high encapsulation efficacy, strong bioadhesion potential and high ex vivo permeation and did not exhibit any deformity to the nasal mucosa. In vitro drug diffusion of optimized formulation showed $92.91 \%$ release of drugs after 180 min. Ex-vivo permeation of drugs across nasal mucosa was $89.03 \%$ after $180 \mathrm{~min}$.
\end{abstract}

Conclusion: Hence, the intranasal administration of topiramate using chitosan can be a promising alternative for brain targeting and the treatment of epilepsy.

Keywords: Topiramate, Nanoparticles, Intranasal administration, Mucoadhesion, Epilepsy

(C) 2020 The Authors. Published by Innovare Academic Sciences Pvt Ltd. This is an open access article under the CC BY license (http://creativecommons.org/licenses/by/4.0/) DOI: http://dx.doi.org/10.22159/ijap.2020v12i5.37385. Journal homepage: https://innovareacademics.in/journals/index.php/ijap

\section{INTRODUCTION}

Epilepsy is a chronic neurological disorder characterized by recurrent seizures or periods of unusual behaviour, and sometimes loss of consciousness [1, 2]. Although, oral route of administration is most convenient for the treatment of epilepsy, has an insignificant role in the treatment of acute epileptic attack due to delayed absorption when given orally. Moreover, in emergency cases, parenteral administration is not possible as it requires medical assistance. Thus, an alternative route of drug delivery is highly desirable for effective outcomes $[3,4]$.

Intranasal route provides greater surface area for absorption of drugs along with porous and highly vascularised epithelial layer. The total blood flow to the nasal site is also greater that facilitates faster drug absorption and hence faster onset of action. Beside this intranasal administration also facilitates the transport of centrally acting drugs through the olfactory region and trigeminal nerves deliver drugs rapidly and efficiently to the brain [5]. Hence it is a convenient and suitable route of administration in the treatment of epilepsy [6]

On the other hand, mucociliary clearance is the limiting factor that decreases the residing time of drug in the nasal cavity, thereby limits the extent of drug absorption [7]. The limitation of mucociliary clearance can be overcome by formulating a dosage form with a mucoadhesive agent. Adhesion of formulation to the mucosal membrane will prevent the drug from early removal out of the nasal cavity and will provide greater time for its absorption to produce a sufficient therapeutic level $[8,9]$.

In this context chitosan, a natural polysaccharide having a biodegradable, biocompatible, non-toxic and mucoadhesive property is been chosen as the carrier for the preparation of mucoadhesive nanoparticles [10]. Besides, it also enhances the paracellular absorption by opening the tight junctions between the epithelial cells [11]. Chitosan, being cationic in nature interacts electrostatically with negatively charged sialic acid moieties on the mucosal membrane, is responsible for its mucoadhesive property [12]. The nanoparticles based formulation provides drug release specifically to the site with improved absorption and activity. Besides, site-specific delivery reduces the side effects of drugs associated with conventional therapy $[13,14]$. Intranasal formulation of antiepileptic agents with chitosan has successfully improved the mucoadhesion, release, and absorption of drugs such as lamotrigine and pregabalin $[15,16]$. Topiramate is a second-generation antiepileptic drug used in partial and generalized seizures. It is used as a prophylactic against migraine headaches, psychiatric disorders, and obesity. Topiramate is also used in children for the treatment of refractory partial seizures with or without secondary generalized tonic-clonic seizures $[17,18]$. Topiramate is only available in oral dosage form and no intranasal formulation is been studied (Product information: TOPAMAXTM. Titusville: Janssen Pharmaceuticals, Inc.; 2011). Administration of Topiramate with food decreases its rate of absorption and it undergoes cytochrome p450 mediated oxidation $[19,20]$. In view of this, the present work was designed to formulate bioadhesive nanoparticles of topiramate by ionotropic gelation method with chitosan and to carry out its characterization. Prepared nanoparticles were evaluated for ex-vivo bioadhesion and permeation using sheep nasal mucosa.

\section{MATERIALS AND METHODS}

\section{Materials}

Chitosan was purchased from Loba Chemie, Mumbai. Topiramate was procured from Johnson and Johnson Pharmaceutical Ltd. Mumbai. Acetic acid was obtained from Fisher Scientific, Mumbai and sodium tripolyphosphate from Hi-Media Laboratories, Mumbai. All other reagents used were of analytical reagent grade.

Preparation of bioadhesive nanoparticles by ionotropic gelation method

Mucoadhesive nanoparticles of topiramate were formulated using chitosan and sodium tri-polyphosphate as a crosslinking agent by 
the ionotropic gelation method. Weighed amount of chitosan and drug was dissolved in $2 \%$ aqueous acetic acid. To it weighed quantity of topiramate was dissolved. Subsequently, $50 \mathrm{ml}$ of the polymer solution was added dropwise to the crosslinking solution with magnetic stirring $(100 \mathrm{rpm})$ at room temperature. The prepared nanoparticles were separated after $30 \mathrm{~min}$, washed with deionized water and subsequently dried at $60{ }^{\circ} \mathrm{C}$ for $3 \mathrm{~h}$ [21]. The composition of different formulations is shown in table 1.

Table 1: Composition of topiramate loaded chitosan nanoparticles

\begin{tabular}{llll}
\hline Formulation code & Topiramate (mg) & Chitosan (mg) & STPP (\%) \\
F1 & 100 & 200 & 2 \\
F2 & 100 & 200 & 3 \\
F3 & 100 & 200 & 4 \\
F4 & 100 & 200 & 5 \\
F5 & 100 & 225 & 4 \\
F6 & 100 & 250 & 4 \\
F7 & 100 & 275 & 4 \\
F8 & 100 & 300 & 4 \\
\hline
\end{tabular}

\section{Characterization of nanoparticles}

\section{Particle size and zeta potential}

The optical microscopy method (Motic BMWB microscope) was used for evaluating the size and shape of the prepared nanoparticles. A small quantity of nanoparticles was dispersed on the slide with the help of a small glass rod. The diameters were determined using a suitable objective $(10 \mathrm{X}, 40 \mathrm{X}, 100 \mathrm{X})$. The average particle size of nanoparticles can be given by the following formula [22].

$$
\text { Average Size }=\frac{\sum \mathbf{n d}}{\sum \mathbf{n}}
$$

Where $\mathrm{n}$ is the number of nanoparticles and $\mathrm{d}$ is the size NP.

Zeta potential determination (surface electric charge) of the prepared nanoparticles was carried out by photon correlation spectroscopy (PCS) using zeta sizer (Malvern Instruments Ltd, Ver. 6.2 ) at $25^{\circ} \mathrm{C}$ and a scattering angle of $90^{\circ} \mathrm{C}$, maintaining electric field strength of $25 \mathrm{Vm}^{-1}$. Briefly, weighed amount of nanoparticles ( $5 \mathrm{mg}$ ) were dispersed in $10 \mathrm{ml}$ of bidistilled water to get optimum $100-200$ kilo-counts $\mathrm{s}^{-1}(\mathrm{kcps})$ for measurement of zeta potential in the range of $+200 \mathrm{mv}$ to- $200 \mathrm{mv}$ [23].

\section{Drug content and entrapment efficiency}

The amount of topiramate incorporated into the nanoparticles was estimated to determine the drug content and entrapment efficiency of the prepared formulations. Nanoparticles equivalent to $10 \mathrm{mg}$ of the topiramate were dissolved in $20 \mathrm{ml}$ of $\mathrm{pH} 6.8$ phosphate buffer solution and kept overnight to extract the drug from the formulation. The solution was then centrifuged $(560 \mathrm{rpm})$ for 10 min to remove the insoluble content. To the obtained solution, $1 \mathrm{ml}$ of ninhydrin solution was added, incubated for $20 \mathrm{~min}$ at $70{ }^{\circ} \mathrm{C}$ and analyzed spectrophotometrically (Analytical Technologies Ltd., Gujarat, India) for drug content at $260 \mathrm{~nm}$. The data was collected by repeating the procedure in triplicate. Given formula was used for calculating drug content entrapment efficiency [24].

$$
\text { Drug content } \%=\frac{\mathrm{Qp}}{\mathrm{Q}} \times 100
$$

Where $Q p=$ quantity of drug encapsulated in nanoparticles

$\mathrm{Q}=$ weighed quantity of powder of nanoparticles

$$
\text { Percent Encapsulation }(E)=\frac{Q p}{Q t} \times 100
$$

Where $\mathrm{E}=$ percentage of encapsulation of nanoparticles

$\mathrm{Qp}=$ quantity of drug encapsulated in nanoparticles

$\mathrm{Qt}=$ quantity of the drug added for encapsulation

\section{Scanning electron microscopy (SEM)}

To study the shape and surface morphology of prepared topiramate nanoparticles, scanning electron microscopy (SEM, JEOL Model JSM$6390 \mathrm{LV}$ ) was used. The samples were mounted on a double- adhesive tape stuck to an aluminum stub. The stubs were coated with gold to a thickness of $\sim 300 \mathrm{~A}^{\circ}$ under an argon atmosphere using a gold sputter module in a high-vacuum evaporator. The coated samples were then randomly scanned and photomicrographs were obtained with SEM [22].

\section{Ex-vivo bioadhesion studies}

The ex vivo bioadhesion property of nanoparticles was performed on a fresh sheep nasal mucosa. Freshly excised sheep nasal mucosa was procured from the local slaughterhouse, cleaned with isotonic saline solution and cut into a piece of $2 \mathrm{~cm}^{2}$ for the study. The topiramate nanoparticles (100 $\mathrm{mg}$ ) were applied on the mucosal surface and attached on a polyethylene plate. Simulated nasal electrolyte solution $(100 \mathrm{ml})$ was poured on nanoparticles. Further, it was incubated in desiccators at $90 \%$ relative humidity for $15 \mathrm{~min}$ so as the polymer interact with the membrane. Further, the plate was kept at an angle of $45^{\circ}$ relative to the horizontal plane. Phosphate buffer $\mathrm{pH} 6.8\left(37^{\circ} \mathrm{C}\right)$ was peristaltically pumped $(5$ $\mathrm{ml} / \mathrm{min}$ ) over the tissue and the perfusate was collected. The amount of topiramate in the collected perfusate was estimated after 1 hour spectrophotometrically. The amount of nanoparticles corresponding to the amount of drug in the perfusate was determined. The amount of adhered nanoparticles was calculated as the difference between the amount of nanoparticles applied and the amount of nanoparticles washed off with perfusate. The percent mucoadhesion was determined by the given equation [15],

$$
\text { Mucoadhesion potential }=\frac{[\text { Concentration of adhered NP }]}{[\text { Concentration of applied NP }]} \times 100
$$

\section{Fourier-transform infrared spectroscopy (FTIR)}

The compatibility studies between topiramate and chitosan were carried out by FTIR (Thermo Nicolet, Avatar 370) to detect drug-excipient interactions if any. Approximately $2 \mathrm{mg}$ sample was powdered uniformly with $200 \mathrm{mg}$ of $\mathrm{KBr}$ for the production of $\mathrm{KBr}$ compacts. The samples were triturated first and then mixed thoroughly with $\mathrm{KBr}$ in 1:1 (sample: $\mathrm{KBr}$ ) ratio, $\mathrm{KBr}$ discs were prepared by compressing the powders at a pressure of 5 tons for $5 \mathrm{~min}$ in a hydraulic press. Scans were obtained at a resolution of $4 \mathrm{~cm}^{-1}$ from 4000 to $400 \mathrm{~cm}^{-1}$. The IR spectra of the physical mixture of the topiramate and chitosan were compared with the spectrum of topiramate and chitosan to determine the compatibility of drug and excipient [24].

\section{Differential Scanning Calorimetry (DSC)}

The thermal behavior of topiramate, chitosan, physical mixture of topiramate and chitosan and prepared topiramate nanoparticles was studied using differential scanning calorimetry (Mettler Toledo DSC 822e) at a rate of $10{ }^{\circ} \mathrm{C} \mathrm{min}{ }^{-1}$ from $30^{\circ}$ to $300{ }^{\circ} \mathrm{C}$ using a nitrogen purge of $50 \mathrm{ml} / \mathrm{min}$ [23].

\section{$\mathrm{X}$-ray diffraction studies (XRD)}

The crystallinities of topiramate, chitosan, physical mixture of topiramate and chitosan and prepared topiramate nanoparticles were analyzed by x-ray diffractometer (Bruker AXS D8 Advance 
diffractometer) with a radius of $240 \mathrm{~mm}$. The pattern was collected with $40 \mathrm{kV}$ of tube voltage and $30 \mathrm{~mA}$ of tube current and scanned over the $2 \theta$ range of $3^{\circ}$ to $80^{\circ}$

\section{In vitro drug diffusion studies}

The drug diffusion characteristic was evaluated with the help of Franz diffusion cell with receptor capacity of $12.0 \mathrm{ml}$ and permeation area of $3.14 \mathrm{~cm}^{2}$. A dialysis membrane $(0.65 \mu \mathrm{m}$ and mol. Wt. of 12000-14000) as a diffusion barrier was used in the donor compartment as a diffusion barrier. The membrane was equilibrated overnight before dispersing the formulation. The temperature of the receptor phase ( $\mathrm{pH} 6.6$ phosphate buffers) was maintained at $37 \pm 1{ }^{\circ} \mathrm{C}$ throughout the experiment by continuous stirring. Nanoparticles equivalent to $10 \mathrm{mg}$ topiramate was placed in the donor compartment. $300 \mu \mathrm{l}$ of the sample was withdrawn at a predetermined time interval from the receiver compartment and the same amount of fresh buffer solution was replaced to maintain the sink conditions. Each experiment was performed in triplicate and the sample was analyzed at a wavelength of $260 \mathrm{~nm}$ spectrophotometrically, taking phosphate buffer ( $\mathrm{pH}$ 6.6) as a blank [23].

\section{Kinetics and mechanism of drug release}

The data obtained from in vitro drug diffusion studies were plotted in various kinetic models: Zero-order, first-order, Higuchi's model and Korsmeyer-Peppas model to study the release kinetics of optimized formulation.

\section{Ex-vivo permeation studies}

Ex vivo permeation study was performed for the optimized batch using freshly excised sheep nasal mucosa, as biomembrane obtained from the local slaughterhouse within $1 \mathrm{~h}$ of sacrificing the animal. Nasal mucosa was carefully cut and mounted on the diffusion chamber with mucosal and serosal surfaces facing towards donor and receptor compartments, respectively. Other experimental procedures of sample collection and analysis were performed in the same way as in for in vitro diffusion studies [25].

\section{Ex-vivo biocompatibility studies}

To determine the biocompatibility of prepared nanoparticles with nasal mucosa. histological study was carried out using freshly excised sheep nasal mucosa, thoroughly cleaned with isotonic saline solution Prepared topiramate nanoparticles $(100 \mathrm{mg})$ were applied properly on the nasal mucosa. After $1 \mathrm{~h}$ it was fixed in $10 \%$ neutral carbonate buffered formalin solution, routinely processed and embedded in paraffin. The experiment was carried out in a cell culture incubator (Sanyo Incubator, Model MCO-5AC, and Japan) to assure optimal conditions for the viability of the tissue. Further paraffin sections $(7.5$ $\mu \mathrm{m})$ were stained with Haematoxylin-Eosin (HE) indicator and observed under the Motic microscope. The untreated mucosa directly fixed after isolation was used as a control [26].

\section{RESULTS AND DISCUSSION}

Physicochemical characterization

Particle size and zeta potential

The percentage yield was calculated to determine the yield of nanoparticles obtained by the ionotropic gelation method. The results of percentage yield, drug content and entrapment efficiency are mentioned in table 2. All the batches of topiramate loaded chitosan nanoparticles showed a percentage yield from 72.02 to $83.73 \%$. From the result, it was observed that as the concentration of chitosan was increased, an increase in percentage yield was observed. The particle size of nanoparticles varies with the concentration of chitosan used. At $1 \% \mathrm{w} / \mathrm{v}$ chitosan concentration, the particles of nanoparticles were irregular with mean size $313.5 \mathrm{~nm}$, which increase for nanoparticles prepared with chitosan, respectively. The topiramate loaded chitosan nanoparticles showed a decrease in particle size. The particle size of all the batches was in the range of $313.5 \mathrm{~nm}$. As the volume of sodium tripolyphosphate was increased, a decrease in the particle size of nanoparticles was observed.

Table 2: Percentage yield, average drug content and entrapment efficiency of mucoadhesive topiramate loaded chitosan nanoparticles

\begin{tabular}{llll}
\hline Batch & Percentage yield & Drug content (\%) & Entrapment efficiency (\%) \\
\hline F1 & 73.66 & $29.38 \pm 2.56$ & $75.66 \pm 1.54$ \\
F2 & 75.48 & $37.15 \pm 2.34$ & $85.02 \pm 2.98$ \\
F3 & 82.85 & $35.46 \pm 2.46$ & $82.42 \pm 1.46$ \\
F4 & 72.02 & $37.25 \pm 2.35$ & $88.42 \pm 2.8$ \\
F5 & 69.85 & $36.87 \pm 1.27$ & $86.39 \pm 1.4$ \\
F6 & 68.73 & $34.96 \pm 2.13$ & $82.28 \pm 1.8$ \\
F7 & 83.73 & $39.45 \pm 2.43$ & $90.45 \pm 1.49$ \\
F8 & 80.33 & $28.24 \pm 2.58$ & $72.18 \pm 2.32$ \\
\hline
\end{tabular}

Results are shown as mean \pm SD $(n=3)$

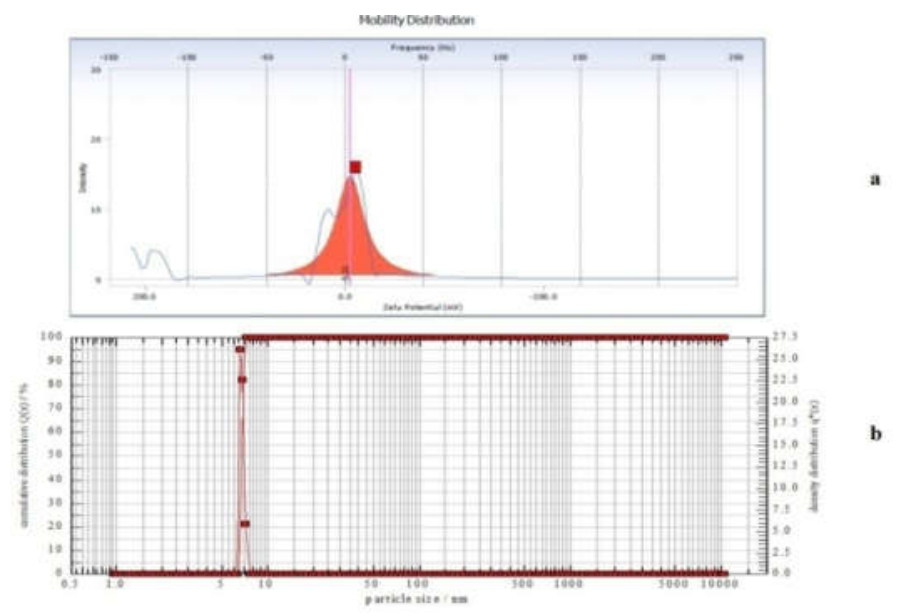

Fig. 1: a. Zeta potential of topiramate loaded chitosan nanoparticles, b. zeta sizer 
The zeta potential of topiramate and chitosan loaded nanoparticles were observed at $-5.21 \mathrm{mv}$ (fig. 1). It showed a negative charge. The formulation displayed negative zeta potential due to the introduction of reduction of droplets size on particles, which increases the anionic property of formulation this permanent negative charge carried by the protonated amino group of the formulation is driving force to the high solubility.

\section{Drug content and entrapment efficiency}

The resultant topiramate loaded chitosan nanoparticles had high drug content and high entrapment efficiency. As shown in table 2, the drug entrapment in the topiramate loaded chitosan nanoparticles was found to be increased with increased polymer: drug ratio. In general, formulations prepared by ionotropic gelation technique demonstrated considerably higher drug encapsulation efficiency. This is also evident from previous studies employing chitosan by the ionotropic gelation technique [27]. It is noteworthy that the F7 batch prepared with $275 \mathrm{mg}$ of chitosan and $4 \%$ of STPP as a crosslinking agent showed the highest entrapment efficiency as $90.45 \%$. Thus, we have considered this formulation for further characterization and ex vivo evaluation.

\section{Scanning electron microscopy (SEM)}

The morphology of prepared nanoparticles was determined by SEM and photomicrographs of topiramate loaded chitosan nanoparticles are displayed in fig. 2. The surface morphology observed by the SEM showed the regular shape and smooth surface area of the prepared nanoparticles. The resultant nanoparticles did not show any ruptures on the surface, validating its rapid clearing from the nasal cavity with a good deposition pattern in the nasal cavity.

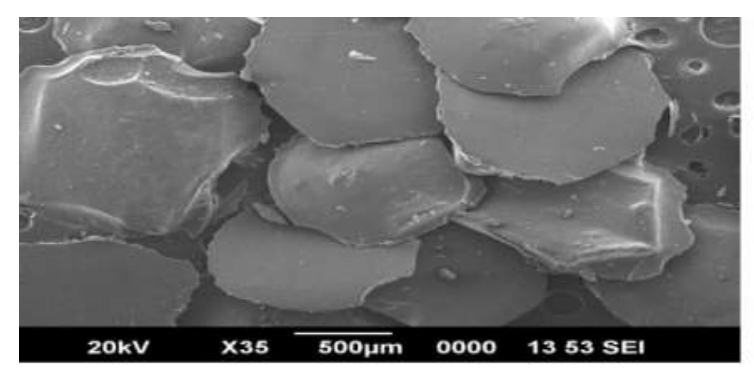

a

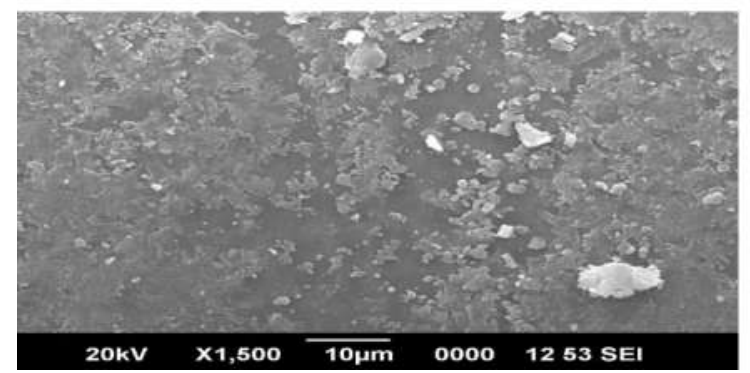

b

Fig. 2: Scanning electron microscopy images of topiramate-loaded chitosan nanoparticles

\section{Ex-vivo bioadhesion studies}

The ex-vivo bioadhesion study was performed on F7 formulation using freshly excised sheep nasal mucosa. The prepared topiramate loaded chitosan nanoparticles showed high bioadhesion strength i.e. $94.30 \%$. The interaction between cationic groups on chitosan and anionic residues such as sialic acid and sulfonic acid on the nasal mucosa might have facilitated the strong adhesion of topiramate loaded chitosan nanoparticles on mucosal surfaces. This is speculated by several earlier findings employing chitosan as a polymer for the preparation of microsphere for nasal drug delivery system.

\section{Fourier-transform infrared spectroscopy (FTIR)}

The FTIR spectra of a mixture of topiramate and chitosan were compared with the spectra of pure chitosan and topiramate for the determination of interaction between drug and excipient. The spectrum of chitosan (fig. 3a.) was characterized by the presence of the band at $2890 \mathrm{~cm}^{-1}$ of $\mathrm{C}-\mathrm{H}$ bonds. The band assigned to $\mathrm{O}-\mathrm{H}$ $\left(3000-3700 \mathrm{~cm}^{-1}\right)$ on the chitosan spectrum. The vibration peak at $3443 \mathrm{~cm}^{-1}$ was indicative of the 0 - $\mathrm{H}$ group. The characteristic bands at $1307 \mathrm{~cm}^{-1}$ and $1091 \mathrm{~cm}^{-1}$ on the chitosan spectrum indicated primary and secondary alcohols. The intense band cantered at 1664 $\mathrm{cm}^{-1}$ on the chitosan spectrum is assigned to the $\mathrm{C}-\mathrm{O}$ bonds of the acetamide groups, referred to as amide band. The obtained FTIR spectra also showed the same absorption peaks as mentioned above confirm the polymer was chitosan. The FTIR spectrum of topiramate (fig. 3b.) showed the characteristics peaks of absorption at 1072.47 $\mathrm{cm}^{-1}\left(\mathrm{~S}=0\right.$ Stretching), $1552.94 \mathrm{~cm}^{-1}(\mathrm{~N}-\mathrm{H}$-stretching) and 1648.25 $\mathrm{cm}^{-1}(\mathrm{C}=0$ stretching). All the absorption peaks are similar as observed in the reference spectra for the drug topiramate (I. P. 2014). The spectrum of topiramate and chitosan physical mixture was characterized by the presence of strong absorption band absorption at $2956.48 \mathrm{~cm}^{-1}\left(\mathrm{C}-\mathrm{H}\right.$ Stretching), $1552.94 \mathrm{~cm}^{-1}(\mathrm{~N}-\mathrm{H}-$ stretching) and $1648.25 \mathrm{~cm}^{-1}(\mathrm{C}=0$ stretching $)$. The observation of the infrared spectroscopy (IR) spectra of the physical mixture of topiramate and polymer chitosan (fig. 3c.) showed all the major peaks of the topiramate, indicating that there was no interaction between the topiramate and chitosan.

\section{Differential scanning calorimetry}

Thermograms of pure topiramate, chitosan, physical mixture of chitosan and topiramate and topiramate-loaded chitosan nanoparticles were shown in fig. 4. DSC thermogram of topiramate illustrated sharp endothermic peak $126.24^{\circ} \mathrm{C}$, due to its melting point. Likewise, the DSC thermogram of chitosan exhibited a broad endothermic peak at $85.36^{\circ} \mathrm{C}$. By investigating the thermogram of topiramate loaded chitosan nanoparticles, (fig. $4 \mathrm{~d}$.) it was found that the endothermic peak corresponding to the melting point of topiramate was found to be reduced in intensity also seemed to be disappeared. This could be attributed to the incorporation and molecular dispersion of topiramate in chitosan polymer matrices of the prepared nanoparticle formulations. 

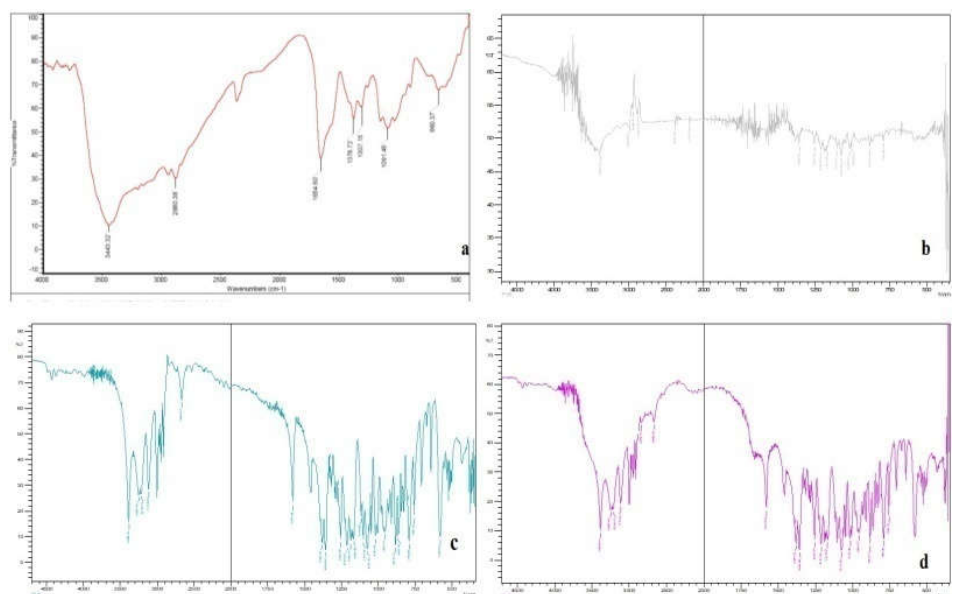

Fig. 3: FTIR spectrum of a. chitosan, b. topiramate, c. a physical mixture of topiramate and chitosan, d. topiramate loaded chitosan nanoparticles

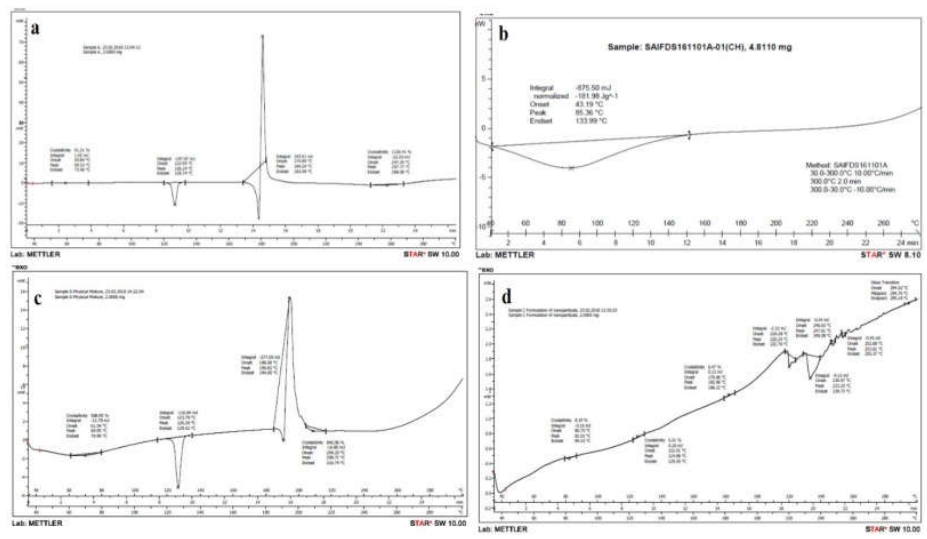

Fig. 4: DSC thermogram of a. Topiramate, b. Chitosan, c. physical mixture of chitosan and topiramate, d. topiramate loaded chitosan nanoparticles
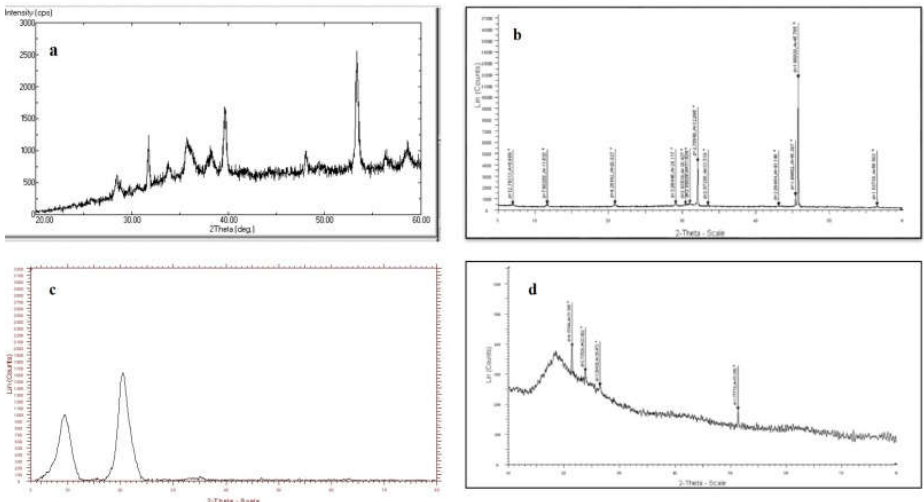

Fig. 5: X-ray diffractogram of a. topiramate, b. chitosan, c. physical mixture of topiramate and chitosan, d. topiramate loaded chitosan nanoparticles

\section{X-ray diffraction studies}

The characteristic XRD spectra of pure topiramate, physical mixture of topiramate and chitosan and topiramate-loaded chitosan-nanoparticles were presented in fig. 5. Characteristic crystalline peaks of topiramate were observed in the pure drug sample reveals the presence of crystalline topiramate. Diffractogram of topiramate loaded chitosan nanoparticles (fig. 5d.) showed the disappearance of crystalline peaks indicating the amorphous form of drugs after its entrapment into chitosan nanoparticles prepared by ionotropic gelation method.

\section{In vitro drug diffusion studies}

The drug release profile of batch F1 to F8 was described in fig. 6 From the graph it was observed that an increase in the chitosan concentration significantly increases the the rate and extent of drug 
release from nanoparticles. A maximum drug release was observed for F7 batch i.e. $94.19 \%$ after $180 \mathrm{~min}$. However, the Release of topiramate from the nanoparticles also changes with the crosslinking of the polymer. An increase in the amount of
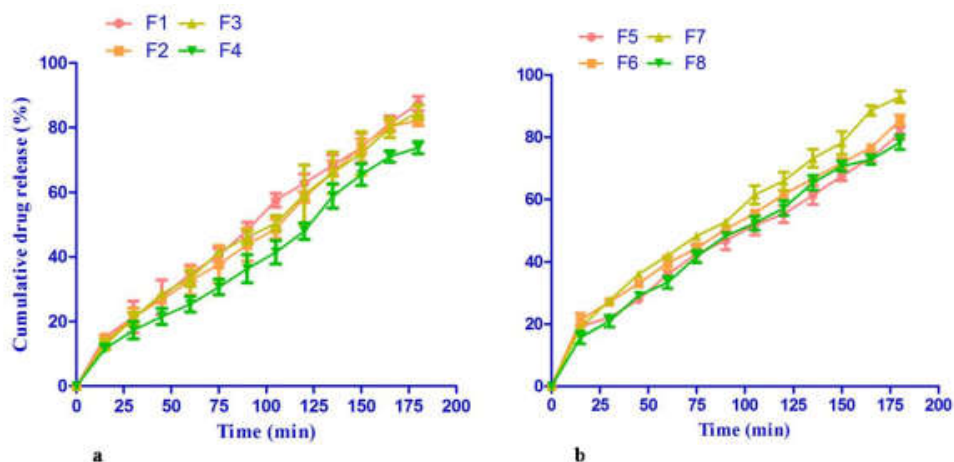

Fig. 6: In vitro drug diffusion study of topiramate loaded chitosan nanoparticles (a: F1 to F4 and b: F5 to F8)

\section{Kinetics and mechanism of drug release}

Results of drug diffusion study of the F7 batch were fitted to kinetic models to investigate the kinetics of drug release (table 3 ). The optimized batch F7 was found to be best fitted in Higuchi kinetic model, as the plot showed highest linearity regression coefficient $\left(\mathrm{R}^{2}\right)$ of 0.9980 compared to First-order (0.9807) and zero-order crosslinking agent reduces the drug release. So the rate of drug release from the nanoparticles prepared by the ionotropic gelation method can be modified by altering the concentration of chitosan and crosslinking agent sodium tripolyphosphate.

Table 3: Release kinetics of topiramate loaded chitosan nanoparticles

\begin{tabular}{lllll}
\hline Formulation code & Zero-order (R) & First order (R) & Higuchi (R) & Korsemeyer peppas (R) (n) \\
\hline F7 & 0.9945 & 0.9807 & 0.9980 & 0.9903 \\
\hline
\end{tabular}

\section{Ex-vivo permeation studies}

The ex-vivo permeation study was carried out for the optimized formulation i.e. F7 based on the results of the in vitro drug diffusion The permeation of topiramate from nanoparticles of batch F7 was found to be $89.03 \%$ after $180 \mathrm{~min}$ as shown in fig. 7. Cationic bioadhesive polymeric material i.e., chitosan, can be used to enhance the drug dissolution and absorption through nanoparticles formulations of drugs for intranasal administration [28]. The Smaller particle size of nanoparticles provides the larger surface area and thus enhances the drug release from the formulations.

\section{Ex-vivo biocompatibility studies}

It is important to maintain nasal mucosal integrity while the preparation of nasal nanoparticles as repeated exposure may lead to the safety issue of the nasal membrane. The drug-loaded nanoparticles treated nasal mucosa did not show any deformity. The results of biocompatibility studies demonstrated that the prepared formulation as safe and biocompatible for intranasal administration. model (0.9945). The release data were also found to be best fitted to Peppas exponential model, to investigate the mechanism of drug release from nanoparticles formulation. The corresponding plot of the Korsmeyer-Peppas model indicated good linearity of regression coefficients $\left(R^{2}=0.9903\right)$. The release exponent $(n)$ was found to be 0.9131. The optimized F7 formulation followed the non-Fickian or anomalous diffusion mechanism of drug release when $n>0.5$.

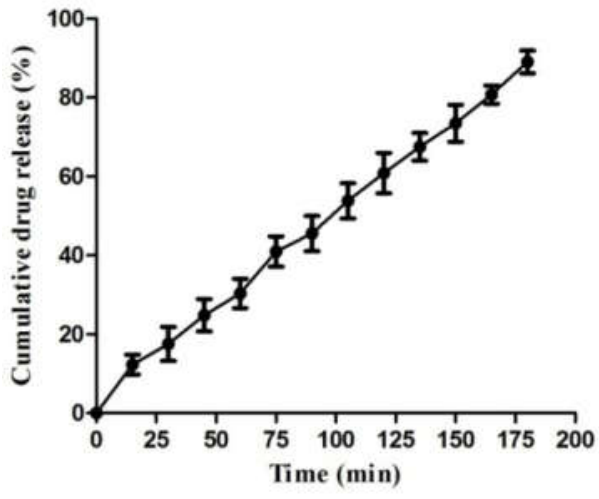

Fig. 7: Ex-vivo permeation of topiramate loaded chitosan nanoparticles of F7 batch

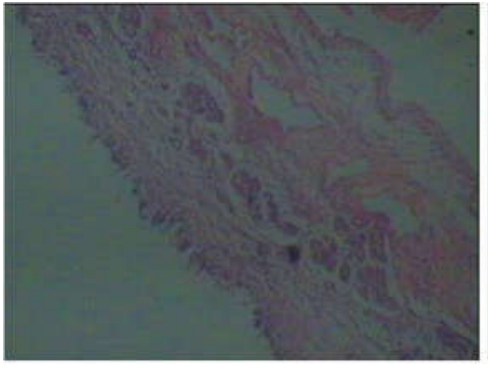

a

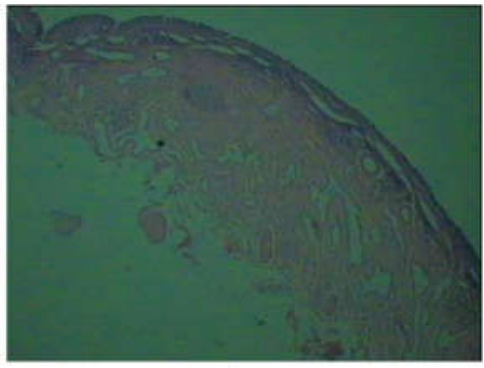

b

Fig. 8: Histopathological specimen of A) untreated nasal mucosa, B) topiramate loaded chitosan nanoparticles treated nasal mucosa 


\section{CONCLUSION}

The present study demonstrated the successful formulation of mucoadhesive nanoparticles of topiramate by ionotropic gelation method. The formulation was characterized by percentage yield, entrapment efficiency, bioadhesion potential, ex vivo biocompatibility, in vitro drug diffusion profile and ex vivo drug permeation study. Physicochemical investigations demonstrated that the topiramate loaded nanoparticles showed the regular shape and smooth surface for nasal administration, high drug content and encapsulation efficacy and showed strong bioadhesion potential without any morphological toxicity to excised sheep nasal mucosa. Also, permeation across excised sheep nasal mucosa exhibited a good permeability of topiramate loaded chitosan nanoparticles. The results of biocompatibility study of topiramate loaded nanoparticles demonstrated that chitosan as safe and biocompatible polymers and can be applied to the nasal epithelium, which could be able to increase the absorption and bioavailability of the drug. Hence, the intranasal administration of topiramate nanoparticles can be considered as the better alternative for administration as compared to its conventional route of administration and dosage form.

\section{FUNDING}

Nil

\section{AUTHORS CONTRIBUTIONS}

All the authors have contributed equally.

\section{CONFLICT OF INTERESTS}

Declared none

\section{REFERENCES}

1. Gautam S, Nikalaje Y, Bhadre D, Trivedi R, Umekar M, Taksande J. Development and evaluation of lamotrigine soya lecithin solid dispersion: in vitro and pharmacodynamic investigation. Int J Appl Pharm 2019;12:115-22.

2. Musumeci T, Bonaccorso A, Puglisi G. Epilepsy disease and nose-to-brain delivery of polymeric nanoparticles: an overview. Pharmaceutics 2019;11:118.

3. Fisher RS, Ho J. Potential new methods for antiepileptic drug delivery. CNS Drugs 2002;16:579-93.

4. Bhuva F, Patel LD, Patel K. Factorial design methodology for development of pediatric nasal spray: study on xylometazoline nasal solution used for treatment of nasal congestion. Indian J Pharm Edu Res 2018;52:218-29.

5. Mantry S, Balaji A. Formulation design and characterization of ropinirole hydrochoride microsphere for intranasal delivery. Asian J Pharm Clin Res 2017;10:195-203.

6. López T, Cuevas JL, Jardon G, Gomez E, Ramirez P. Preparation and characterization of antiepileptic drugs encapsulated in solgel titania nanoparticles as controlled release system. Med Chem 2015;2:3.

7. Tas C, Ozkan CK, Savaser A, Ozkan Y, Tasdemir U, Altunay H. Nasal administration of metoclopramide from different dosage forms: in vitro, ex vivo, and in vivo evaluation. Drug Delivery 2009; 16:167-75

8. Dhanda DS, Frey WH, Leopold D, Kompella UB. Approaches for drug deposition in the human olfactory epithelium. Drug Delivery Technol 2005;5:64-72.

9. Gavini E, Rassu G, Sanna V, Cossu M, Giunchedi P. Mucoadhesive microspheres for nasal administration of an antiemetic drug, metoclopramide: in-vitro/ex-vivo studies. J Pharm Pharmacol 2005;57:287-94.
10. Al-Nemrawi NK, Alsharif SS, Dave RH. Preparation of chitosanTPP nanoparticles: the influence of chitosan polymeric properties and formulation variables. Int J Appl Pharm 2018;10:60-5.

11. Hagesaether E. Permeation modulating properties of natural polymers-effect of molecular weight and mucus. Int J Pharm 2011;409:150-5.

12. Soni M, Majumdar A, Malviya N. Mucoadhesive chitosan microspheres of gefitinib. Int J Curr Pharm Res 2018;10:9-19.

13. Santos Magalhaes NS, Pontes A, Pereira VM, Caetano MN Colloidal carriers for benzathine penicillin G: nanoemulsions and nanocapsules. Int J Pharm 2000;208:71-80.

14. Kulkarni A, Bambole VA, Mahanwar PA. Electrospinning of polymers, their modeling and applications. Polymer Plastics Tech Eng 2010;49:427-41.

15. Taksande JB, Sonwane PP, Trivedi RV, Wadher KJ, Umekar MJ. Formulation and pharmacodynamic investigations of lamotrigine microspheres in pentylenetetrazole-induced seizures in mice. Asian J Pharm 2017;11:S216-24.

16. Taksande JB, Umekar MJ. Preparation of intranasal pregabalin microspheres: in vitro, ex vivo and in vivo pharmacodynamic evaluation. J Pharm Res 2018;12:112-21.

17. El-din EY, Omar AR. Effect of prenatal administration of therapeutic dose of topiramate on placentae albino rats' fetuses. Int J Pharm Pharm Sci 2017;9:54-9.

18. Sommer BR, Mitchell EL, Wroolie TE. Topiramate: effects on cognition in patients with epilepsy, migraine headache and obesity. Ther Adv Neurol Disord 2013;6:211-27.

19. Doose DR, Walker SA, Gisclon LG, Nayak RK. Single-dose pharmacokinetics and effect of food on the bioavailability of topiramate, a novel antiepileptic drug. J Clin Pharmacol 1996;36:884-91.

20. Britzi M, Soback S, Isoherranen N, Rene'H L, Perucca E, Doose $\mathrm{DR}$, et al. Analysis of topiramate and its metabolites in plasma and urine of healthy subjects and patients with epilepsy by use of a novel liquid chromatography-mass spectrometry assay. Ther Drug Monit 2003;25:314-22.

21. Raj BS, Punitha IS, Bodiwala J. Formulation and evaluation of chitosan prazosin beads by ionotropic gelation method. Int J Res Pharm Chem 2012;2:974-83.

22. Belgamwar VS, Patel HS, Joshi AS, Agrawal A, Surana SJ, Tekade AR. Design and development of nasal mucoadhesive microspheres containing tramadol $\mathrm{HCl}$ for CNS targeting. Drug Delivery 2011;18:353-60.

23. Pardeshi CV, Rajput PV, Belgamwar VS, Tekade AR Formulation, optimization and evaluation of spray-dried mucoadhesive microspheres as intranasal carriers for valsartan. J Microencap 2012;29:103-14.

24. Taksande JB, Wadher KJ, Trivedi RV, Umekar MI. Development and evaluation of lamotrigine loaded n-trimethyl chitosan microspheres for intranasal administration. Int J Chem Tech Res 2017;10:1-13.

25. Chalikwar SS, Mene BS, Pardeshi CV, Belgamwar VS, Surana SJ. Self-assembled, chitosan grafted PLGA nanoparticles for intranasal delivery: design, development and ex vivo characterization. Polymer Plastics Technol Eng 2013;52:368-80.

26. Pardeshi CV, Belgamwar VS. Controlled synthesis of $\mathrm{N}, \mathrm{N}, \mathrm{N}-$ trimethyl chitosan for modulated bio adhesion and nasal membrane permeability. Int J Bio Macromol 2016;82:933-44.

27. Ma L, Liu C. Preparation of chitosan microspheres by ionotropic gelation under a high voltage electrostatic field for protein delivery. Colloids Surf B 2010;75:448-53.

28. Tiyaboonchai W. Chitosan nanoparticles: a promising system for drug delivery. Naresuan University J 2013;11:51-66. 